\title{
Is categorization performance really intact in amnesia? A meta-analysis
}

\author{
SAFA R. ZAKI \\ Williams College, Williamstown, Massachusetts
}

\begin{abstract}
Most published studies of category learning in amnesia have reported intact categorization performance. These results have been used to challenge single-system accounts of categorization and recognition, in which a single representational system mediates performance in these two tasks. Many of the published studies, however, have shown a numerical advantage for controls over amnesics and often have had low statistical power. A meta-analysis was conducted to assess whether this numerical advantage is significant when the data are pooled across studies. This analysis indicates that amnesic subjects do, in fact, show deficits in categorization tasks, which is consistent with single-system exemplar model predictions.
\end{abstract}

In categorization research, a fervent debate has surrounded the nature of the representation of categories. Prototype theory assumes that people abstract the central tendency or prototype of a category after exposure to individual instances and subsequently use that summary representation for making categorization decisions (Homa, 1984; Posner \& Keele, 1968; Smith \& Minda, 1998). Conversely, exemplar theorists assume that people store the individual exemplars and use these stored examples as the basis for future categorization decisions (Hintzman, 1986; Medin \& Schaffer, 1978; Nosofsky, 1986).

Because exemplar models assume that specific exemplar information is stored, these models predict a performance relation between categorization and recognition. That is, because categorization decisions are based on stored items, the ability to categorize should be related to the ability to remember particular items. This prediction has received some empirical support from mainstream categorization research (Nosofsky, 1988).

However, in recent years, this assumption has been challenged by findings from the cognitive neuroscience literature. In a number of studies, amnesics were shown to perform poorly on explicit memory tasks, whereas their performance on categorization tasks was comparable to that of a control group (e.g., Knowlton \& Squire, 1993; Reed, Squire, Patalano, Smith, \& Jonides, 1999; Squire \& Knowlton, 1995). A frequently cited article in support of the dissociation between categorization and recognition is Knowlton and Squire's (1993) study. Knowlton and

This research was supported in part by funding from the Williams College Science Center and a McDonnell-Pew Consortium Grant. I thank John Rudoy and Bethany Smith for research assistance, Kris Kirby for helpful discussions, and Rob Nosofsky, Brian Ross, and three anonymous reviewers for comments on an earlier version of this article. Correspondence concerning this article should be addressed to S. R. Zaki, Department of Psychology, Bronfman Science Center, Williams College, Williamstown, MA 01267 (e-mail: szaki@williams.edu).
Squire tested amnesics and matched controls in Posner and Keele's (1968) dot pattern paradigm. In a categorization task, subjects viewed various exemplars of a category on a computer screen and were then asked to categorize various new items that were either members or nonmembers of that category. In the recognition task, the subjects viewed several unrelated items on the screen and were given an old/new recognition test. The key finding was that although the amnesics showed a large deficit in the recognition task, their performance on the categorization task was not statistically different from that of the control group. This evidence was taken as support for the idea that, contrary to the assumption made by exemplar models, separate representational systems underlie categorization and explicit memory. Specifically, an explicit memory system is damaged in amnesics, leading to poor performance on old/new recognition, whereas a procedural system that is responsible for categorization is intact.

This dissociation has been demonstrated in a variety of stimulus domains, such as dot pattern categorization, cartoon animal categorization, artificial grammar learning, and a weather prediction task. For example, Reed et al. (1999) generalized Knowlton and Squire's (1993) findings to line drawings of animals that varied on nine binary-valued dimensions. In a training phase, subjects viewed various low-level distortions of a prototypical cartoon animal. In a test that followed, they were asked to indicate whether or not particular new items were members of the previously viewed category. In addition, the subjects were given an explicit memory test in which they were asked to recall the two possible values of each of the nine dimensions. Although the amnesic patients were significantly impaired at the explicit memory task, they were able to categorize the cartoon animals at levels comparable to those of the control group.

However, single-system accounts of these data sets have also been put forth. Following the exemplar model approach, Nosofsky and Zaki (1998) suggested that both 
categorization and recognition depend on a single representational system that is accessed when either kind of judgment is made. Nosofsky and Zaki argued that a general memory impairment would simply be more detrimental to a recognition task than to a categorization task. The general intuition for this argument is that in categorization, subjects need only have a vague sense of the previously viewed items to complete the task, whereas the recognition task requires that subjects remember the old items in finer detail.

Using two approaches, Nosofsky and Zaki (1998) provided support for the single-system hypothesis. First, by delaying the test phase of the Knowlton and Squire (1993) categorization task, they were able to introduce a memory impairment in college-aged normal subjects. Like amnesia, delay caused a small drop in categorization performance and a substantial drop in recognition performance. In a second approach, Nosofsky and Zaki also modeled Knowlton and Squire's (1993) data, using a formalized exemplar model, the generalized context model (Nosofsky, 1986). In this analysis, Nosofsky and Zaki demonstrated that the model could accurately predict Knowlton and Squire's (1993) data (although see Smith \& Minda, 2001, and Zaki \& Nosofsky, 2004, for a debate regarding the exemplar approach), so long as the memory sensitivity parameter, which is an index of the discriminability of items in memory, was set at a lower level for the amnesics than for the control group. Therefore, Nosofsky and Zaki demonstrated that the Knowlton and Squire (1993) dissociation did not necessarily imply the workings of two separate memory systems.

Similarly, Zaki and Nosofsky (2001) demonstrated that Reed et al.'s (1999) findings involving line drawings of animals were consistent with a single-system account. For the Reed et al. stimuli, the dimensions of the stimuli were highly correlated within the category, so that a participant could literally focus on any one dimension and perform at normal levels in the categorization task (Zaki \& Nosofsky, 2001). However, in the explicit memory task, the participants needed to attend to many of the dimensions in order to perform at normal levels. Zaki and Nosofsky (2001) tested subjects in a delayed test version of the Reed et al. task. Delay, like amnesia, caused a small drop in categorization performance and a large drop in performance on the explicit memory task. In addition, formal modeling analyses demonstrated that the subjects were indeed paying attention to only a few of the stimulus dimensions in the categorization task. Therefore, the dissociation between categorization and explicit memory could be explained in terms of the different task requirements, rather than in terms of separate memory systems.

Although the approach put forth by single-system theorists provides an alternative account to the multiplesystems approach, both models are consistent with the data. There have been recent attempts to test predictions of a single-system model by collecting new data (e.g., Zaki, Nosofsky, Jessup, \& Unverzagt, 2003). However, these attempts have been limited by the availability of amnesic subjects. A potentially fruitful way to test the predictions of the model is to look more closely at the data in the literature. Multiple- and single-system accounts of categorization and recognition make different general predictions regarding how people perform in categorization tasks. According to the single-system model, if the hypothesis of a single representational system is correct, categorization performance should, on average, be at least slightly lower for amnesics than for controls. The model is constrained to predict this deficit because a memory deficit must affect both categorization and recognition judgments. In contrast, according to the multiple-systems account, if categorization and recognition are indeed mediated by separate representational systems and the categorization system in amnesics is intact, no consistent difference is predicted between the categorization performance of amnesics and controls. ${ }^{1}$

The number of studies in which no significant difference has been observed between the performances of amnesics and controls on categorization has been taken as support for the multiple-systems approach (e.g., Filoteo, Maddox, \& Davis, 2001; Knowlton \& Squire, 1993; Reed et al., 1999; Squire \& Knowlton, 1995). However, a perusal of this literature suggests a pattern of results that may be supportive of a single-system account. In many of the studies, the amnesics show a small nonsignificant numerical impairment in the categorization task. Moreover, the sample sizes used in many of these studies have often been relatively small, undoubtedly restricted by the difficulty in locating amnesic patients to test. Power analyses of a number of these studies suggest that they lack the power to detect small, medium, or sometimes even large-sized effects, as defined by Cohen (1988). For example, in Knowlton and Squire's (1993) classic study, 10 amnesics and 12 controls were tested. Given this sample size, the power to detect small, medium, and large effects was $.07, .19$, and .42 , respectively. ${ }^{2}$ These levels are well below the recommended level of .8 (Cohen, 1988). This limited power raises problems because the multiple-systems argument rests on accepting the null hypothesis.

Given the relative rarity of amnesic patients, one way to overcome the lack of power in these previous studies is to pool results across studies. Therefore, the aim of the present study is to conduct a meta-analysis of the results of categorization by amnesics to ascertain whether there is evidence of an impairment in categorization performance across studies. An observed impairment would be in line with the predictions of the single-system account, in which one representational system mediates categorization and recognition.

\section{METHOD}

\section{Literature Search}

The literature search was restricted to published journal articles. Although meta-analyses often include unpub- 
lished studies, they do so to combat the bias to selectively publish only those studies with significant results (Rosenthal, 1995). However, because the focus of the reported studies is on the null results in the categorization task and positive results in the explicit memory task, there appears to be little risk of a publication bias. To search the published literature, computer-based searches were conducted on PsycLit and MedLine with key words such as categorization and classification paired with amnesia and amnesic. In addition, the references in relevant articles were searched for further articles of interest. Once the articles had been identified, the following criteria were used to select the articles for inclusion in the metaanalysis.

\section{Inclusion Criteria}

All the studies selected for inclusion had to be published in peer-reviewed English-language journals. Critically, the studies had to compare the performance of people with amnesia with that of healthy controls on a categorylearning task. A category-learning task was defined as a task in which subjects learned one or more categories of stimuli. Category-learning tasks that met these criteria included probabilistic category learning, dot pattern categorization, classification of painting style, verbal category learning, and artificial grammar learning. Finally, sufficient data had to be reported in the article for its inclusion in the meta-analysis. Two studies were excluded because, despite the fact that they met the criteria above, the data had been previously published (at least in part) in other articles (Knowlton, Mangels, \& Squire, 1996; Squire \& Zola, 1996). In addition, one study was excluded because of the potential for more widespread cognitive deficits in the patient group (Zaki et al., 2003).

\section{Meta-Analytic Procedure}

Two main meta-analytic procedures with different strengths were used in the present study. The first approach was to use a vote-counting procedure in which the direction of the effect across studies was tallied and compared with chance (Bushman, 1994). One major drawback of the vote-counting procedure is that it ignores information regarding the sample size and the effect size of the studies. Therefore, a more formal meta-analysis in which the effect sizes were weighted by the sample size was also conducted (Rosenthal, 1991). This approach is limited to the extent that the data are unavailable from the full set of studies. Thus, the two approaches were used to provide complementary techniques.

\section{RESULTS}

The literature search revealed 12 articles that met the criteria for inclusion in the study. Articles preceded by an asterisk in the reference section were included in the analysis. Table 1 summarizes the observed means for the categorization tasks in these articles. Although in several cases single published reports are represented by more than one mean in the table (e.g., in the case of mul- tiple experiments), only one mean was entered into the formal analysis, so as not to violate assumptions of independence. ${ }^{3}$ There were two exceptions to this rule (Knowlton \& Squire, 1994; Knowlton, Squire, \& Gluck, 1994). In both of these cases, two experiments within a single published report yielded effects in different directions. For example, in the first experiment of Knowlton and Squire's (1994) article, the data yielded a slight advantage for the amnesic group, whereas the reverse was true for the second experiment. Because the results were qualitatively different, the two experiments were not combined. 4

The first analysis conducted was the vote-counting procedure. For each study, if the categorization score for the controls was numerically greater than the categorization score for the amnesics, that result was considered a positive one. The positive results are indicated in Table 1. The results indicate that the controls scored better than the amnesics 12 out of 14 times. A sign test was used to determine whether the proportion of positive outcomes differed significantly from .5. The rationale for this test is that if there are no differences in the performance of the amnesics and the controls in these tasks, the amnesics should perform better than the controls about half of the time. A binomial test indicated that the controls performed at significantly higher levels than the amnesics $(p=.01)$.

For the second analysis, an effect size was calculated from means and standard deviations, $t$ statistics, or $F$ statistics, for each study that provided the necessary information. ${ }^{5}$ The standardized mean difference $d$ (Cohen, 1988) was used as a measure of effect size. In two of the studies (Filoteo et al. 2001; Knowlton et al., 1994) used in the previous analysis, insufficient information was available to calculate the effect size, and so these studies are not included in the present analysis. In addition, Squire and Knowlton's (1995) study was not included, because only one patient was tested.

Once again, only one outcome per published report was entered into the analysis. In the case of multiple experiments per report, the results were meta-analytically combined into one effect size. There were two exceptions to this rule. One exception was Knowlton and Squire's (1994) study, mentioned in the previous analysis. The other was Kolodny's (1994) study. In the two experiments in this article, two different types of categorization were tested. The task in which participants classified artistic style yielded a strong and significant advantage for the control group. The categorization of dot patterns yielded a milder positive effect. Both were entered into the metaanalysis so that they could be separated in the subsequent analysis discussed below.

The effect sizes are plotted with 95\% confidence intervals in Figure 1. The effect sizes were positive in all but one case (one of two experiments in Knowlton \& Squire, 1994). For significance testing, a random effects model was chosen because of the likely heterogeneous nature of the data set. The random effects model yielded a combined effect size of $d=.62, p<.001$ (two-tailed test). 


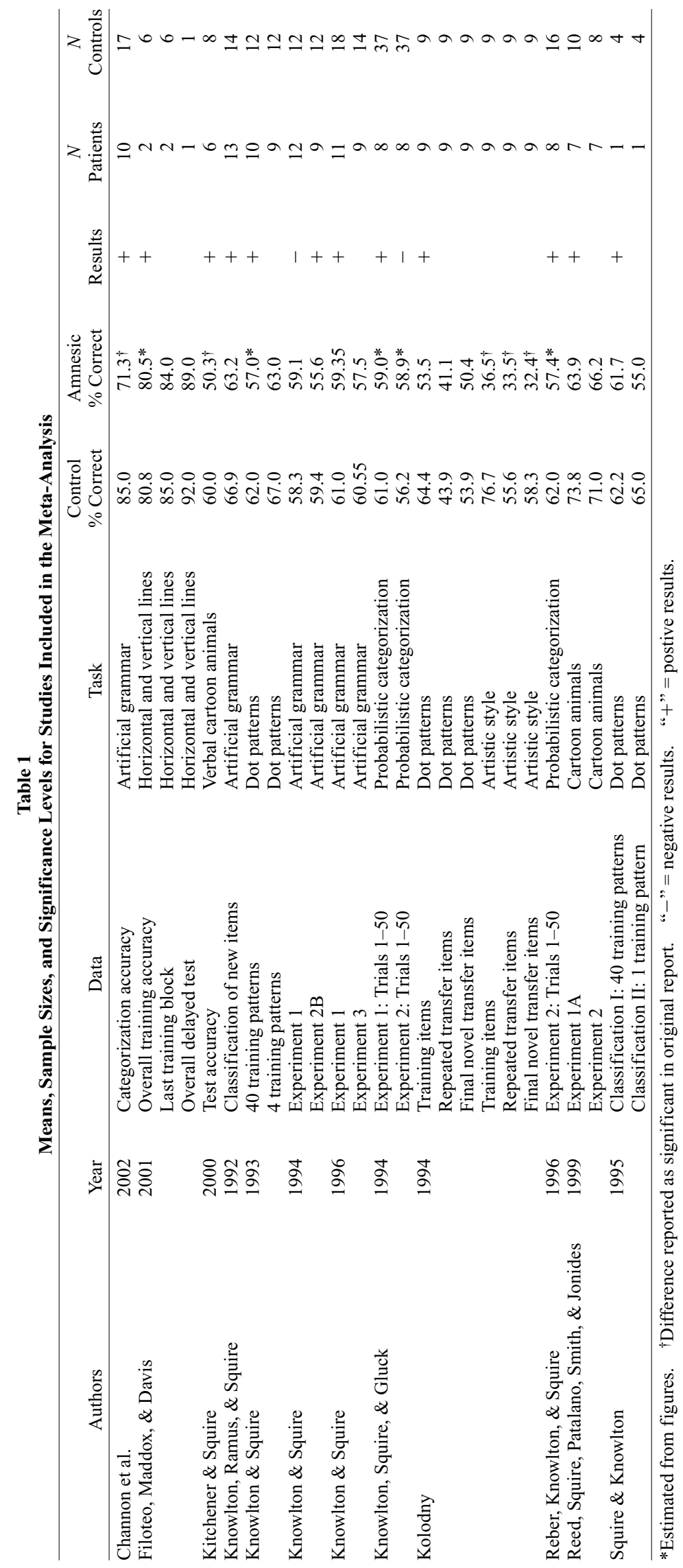




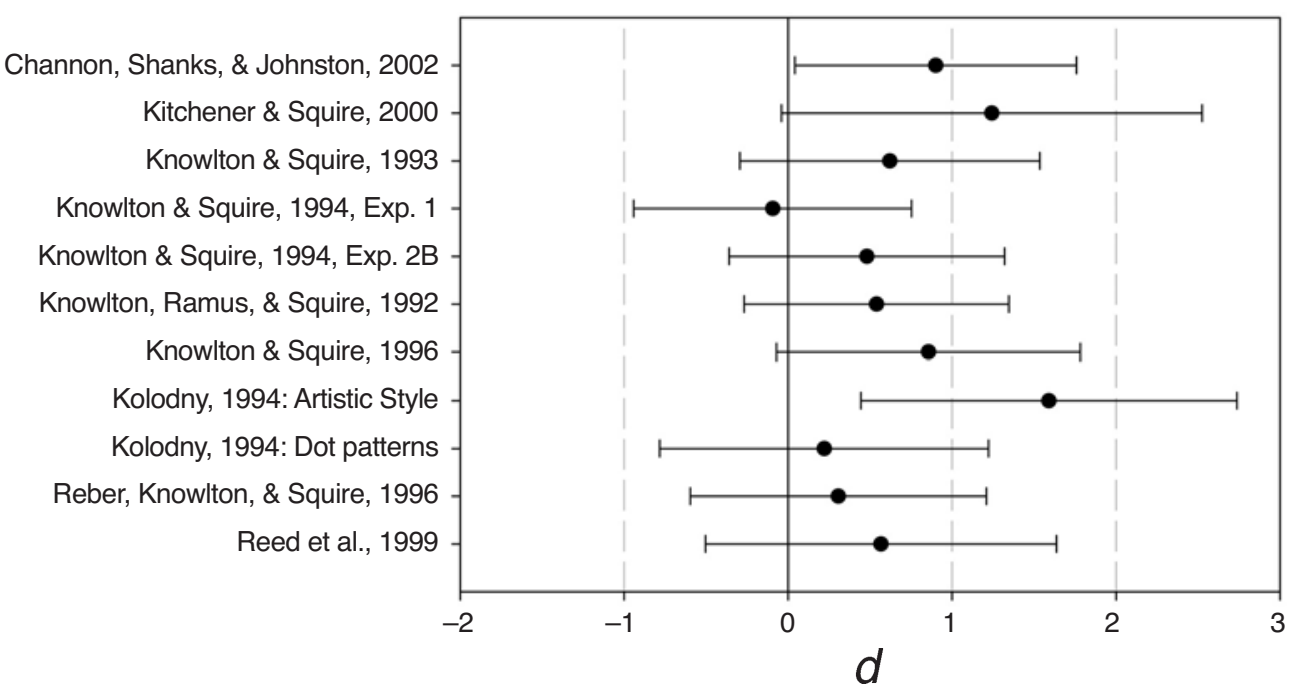

Figure 1. Plot of the effect sizes of each study entered into the meta-analysis. The mean $\boldsymbol{d}$ for each study is represented by the filled circles, and the horizontal lines indicate the $95 \%$ confidence intervals.

One potential criticism of this analysis is that it combines effects in the literature that are known to be significant with others that are nonsignificant. One question that arises is whether the combined effect size is significant only because of the inclusion of these stronger findings. Multiple-systems theorists have sometimes given post hoc explanations for these significant findings, and so from the multiple-systems perspective, they may reflect different sorts of processing than do the other categorization results. For example, Kitchener and Squire (2000) demonstrated that amnesics are impaired at categorizing the Reed et al. (1999) stimuli when these stimuli are verbally presented to the subjects. Kitchener and Squire argued that the verbal presentation of the stimuli makes demands on declarative memory that causes a performance deficit in amnesia. To test whether the significance of the previous meta-analysis was due to the inclusion of these stronger effects, I conducted another analysis in which the significant findings were removed from the set of outcomes used. The combined effect size for the included studies was $d=.44, p<.01$. This result suggests that the categorization impairment in amnesics is not limited to tasks in which significant impairment has been previously observed.

In conclusion, the various analyses conducted provide converging evidence that categorization performance is, in fact, impaired in amnesia.

\section{DISCUSSION}

According to the multiple-systems approach, categorization is mediated by a procedural system that is intact in amnesia, and recognition and recall are mediated by a declarative system that is damaged in amnesia. Reports of dissociations between categorization and recognition in amnesics have been used to support this model. In these studies, amnesics show large deficits in explicit memory tasks but perform at near-normal levels in categorization tasks.

However, these dissociations have also been explained by a single-system account in which a single representational system is responsible for both categorization and recognition. Indeed, in the experimental paradigms that have been tested, the single-system exemplar model generally predicts very small quantitative differences between the categorization performances of amnesics and normal controls. The results of this meta-analysis are, therefore, consistent with the predictions of single-system models.

\section{Alternative Accounts and Future Directions}

Although the multiple-systems account of categorization and recognition does not make an a priori prediction of a deficit in categorization in amnesia, it can account for the results of the meta-analysis by making certain assumptions about the nature of the tasks, the nature of the brain deficit, or the interaction of the separate systems. For example, it is possible to postulate that the normal controls use declarative memory to some extent in these categorization tasks and that the categorization deficit in amnesia reflects the amnesics' inability to use their declarative memory. Another possibility is to argue that the brain damage in the amnesics tested is not always limited to the areas that govern declarative memory but may extend, in some part, to categorization areas as well.

Aside from separate systems for categorization and recognition, researchers have recently argued for the existence of separate systems that are responsible for different types of categorization behavior (e.g., Ashby, AlfonsoReese, Turken, \& Waldron 1998; Erickson \& Kruschke, 1998; Maddox, Ashby, \& Bohil, 2003). For example, Ashby, Maddox, and colleagues have argued that the learning of different types of category structures - namely, rule- 
based and information integration category learning - is mediated by different brain systems (e.g., Ashby, Ell, \& Waldron, 2003; Maddox, Bohil, \& Ing, 2004). In addition, recent neuroimaging studies (Reber, Gitelman, Parrish, \& Mesulam, 2003) suggest that different areas of the brain might be involved in explicit versus implicit category learning. Single-system researchers will need to address these extant challenges. Nonetheless, with the demonstration of a categorization impairment in amnesia, the present analysis removes the challenge posed to the single-system approach by apparent dissociations between categorization and recognition.

\section{REFERENCES}

References marked with an asterisk indicate studies included in the meta-analysis.

Ashby, F. G., Alfonso-Reese, L. A., Turken, A. U., \& Waldron, E. M. (1998). A neuropsychological theory of multiple systems in category learning. Psychological Review, 105, 442-481.

Ashby, F. G., Ell, S. W., \& WALDron, E. M. (2003). Procedural learning in perceptual categorization. Memory \& Cognition, 31, 11141125 .

Bushman, B. J. (1994). Vote-counting procedures in meta-analysis. In H. Cooper \& L. V. Hedges (Eds.), The handbook of research synthesis (pp. 193-214). New York: Russell Sage Foundation.

*Channon, S., Shanks, D., Johnstone, T., Vakili, K., Chin, J., \& SinCLAIR, E. (2002). Is implicit learning spared in amnesia? Rule abstraction and item familiarity in artificial grammar learning. Neuropsychologia, 40, 2185-2197.

COHEN, J. (1988). Statistical power analysis for the behavioral sciences (2nd ed.). Hillsdale, NJ: Erlbaum.

Erickson, M. A., \& KruschKe, J. K. (1998). Rules and exemplars in category learning. Journal of Experimental Psychology: General, 127, 107-140.

*Filoteo, J. V., Maddox, W. T., \& Davis, J. D. (2001). Quantitative modeling of category learning in amnesic patients. Journal of the International Neuropsychological Society, 7, 1-19.

HinTZMAN, D. L. (1986). "Schema abstraction" in a multiple-trace memory model. Psychological Review, 93, 411-428.

Hома, D. (1984). On the nature of categories. In G. H. Bower (Ed.), The psychology of learning and motivation: Advances in research and theory (Vol. 18, pp. 49-94). San Diego: Academic Press.

*Kitchener, E. G., \& SQuire, L. R. (2000). Impaired verbal category learning in amnesia. Behavioral Neuroscience, 114, 907-911.

Knowlton, B. J., Mangels, J. A., \& SQuiRe, L. R. (1996). A neostriatal habit learning system in humans. Science, 273, 1399-1402.

*Knowlton, B. J., Ramus, S. J., \& SQuire, L. R. (1992). Intact artificial grammar learning in amnesia: Dissociation of classification learning and explicit memory for specific instances. Psychological Science, 3, 172-179.

* KNOWLTON, B. J., \& S Quire, L. R. (1993). The learning of categories: Parallel brain systems for item memory and category knowledge. Science, 262, 1747-1749.

* KNOWLTON, B. J., \& SQuire, L. R. (1994). The information acquired during artificial grammar learning. Journal of Experimental Psychology: Learning, Memory, \& Cognition, 20, 79-91.

*Knowlton, B. J., \& SQuire, L. R. (1996). Artificial grammar learning depends on implicit acquisition of both abstract and exemplarspecific information. Journal of Experimental Psychology: Learning, Memory, \& Cognition, 22, 169-181.

* KnOwlton, B. J., Seuire, L. R., \& GluCK, M. A. (1994). Probabilistic classification learning in amnesia. Learning \& Memory, 1, 106-120.

*Kolodny, J. A. (1994). Memory processes in classification learning: An investigation of amnesic performance in categorization of dot patterns and artistic styles. Psychological Science, $\mathbf{5}$, 164-169.
Maddox, W. T., Ashby, F. G., \& Bohil, C. J. (2003). Delayed feedback effects on rule-based and information-integration category learning. Journal of Experimental Psychology: Learning, Memory, \& Cognition, 29, 650-662.

MADDOX, W. T., Bohil, C. J., \& ING, A. D. (2004). Evidence for a procedural-learning-based system in perceptual category learning. Psychonomic Bulletin \& Review, 11, 945-952.

MATT, G. E., \& CoOK, T. D. (1994). Threats to the validity of research synthesis. In H. Cooper \& L. V. Hedges (Eds.), The handbook of research synthesis (pp. 503-520). New York: Russell Sage Foundation.

Medin, D. L., \& Schaffer, M. M. (1978). Context theory of classification learning. Psychological Review, 85, 207-238.

Nosofsky, R. M. (1986). Attention, similarity, and the identificationcategorization relationship. Journal of Experimental Psychology: General, 115, 39-57.

NosOFSKY, R. M. (1988). Exemplar-based accounts of relations between classification, recognition, and typicality. Journal of Experimental Psychology: Learning, Memory, \& Cognition, 14, 700-708.

NosofsKy, R. M., \& ZAKI, S. R. (1998). Dissociations between categorization and recognition in amnesic and normal individuals: An exemplar-based interpretation. Psychological Science, 9, 247-255.

Posner, M. I., \& Keele, S. W. (1968). On the genesis of abstract ideas. Journal of Experimental Psychology, 77, 353-363.

Reber, P. J., Gitelman, D. R., Parrish, T. B., \& Mesulam, M. M. (2003). Dissociating explicit and implicit category knowledge with fMRI. Journal of Cognitive Neuroscience, 15, 574-583.

*Reber, P. J., Knowlton, B. J., \& Squire, L. R. (1996). Dissociable properties of memory systems: Differences in the flexibility of declarative and nondeclarative knowledge. Behavioral Neuroscience, 110, 861-871.

*ReD, J. M., SQuire, L. R., Patalano, A. L., Smith, E. E., \& Jonides, J. (1999). Learning about categories that are defined by object-like stimuli despite impaired declarative memory. Behavioral Neuroscience, 113, 411-419.

RoSENTHAL, R. (1991). Meta-analytic procedures for social research (Rev. ed.). Thousand Oaks, CA: Sage.

Rosenthal, R. (1995). Writing meta-analytic reviews. Psychological Bulletin, 118, 183-192.

Smith, J. D., \& Minda, J. P. (1998). Prototypes in the mist: The early epochs of category learning. Journal of Experimental Psychology: Learning, Memory, \& Cognition, 24, 1411-1436.

SMITH, J. D., \& MindA, J. P. (2001). Journey to the center of the category: The dissociation in amnesia between categorization and recognition. Journal of Experimental Psychology: Learning, Memory, and Cognition, 27, 984-1002.

*SQUIRE, L. R., \& KNOWLTON, B. J. (1995). Learning about categories in the absence of memory. Proceedings of the National Academy of Sciences, 92, 12470-12474.

SQUIRE, L. R., \& ZoLA, S. M. (1996). Structure and function of declarative and nondeclarative memory systems. Proceedings of the $\mathrm{Na}$ tional Academy of Sciences, 93, 13515-13522.

ZAKI, S. R., \& NosOFSKY, R. M. (2001). A single-system interpretation of dissociations between recognition and categorization in a task involving object-like stimuli. Cognitive, Affective, \& Behavioral Neuroscience, 1, 344-359.

ZAKI, S. R., \& NOSOFSKY, R. M. (2004). False prototype enhancement effects in dot pattern categorization. Memory \& Cognition, 32, 390398.

Zaki, S. R., Nosofsky, R. M., Jessup, N. M., \& Unverzagt, F. W. (2003). Categorization and recognition performance of a memoryimpaired group: Evidence for single-system models. Journal of the International Neuropsychological Society, 9, 394-406.

\section{NOTES}

1. As will be addressed further in the discussion, multiple-systems theorists may be able to account for an observed categorization impairment in amnesia by assuming, for example, that brain damage in these amnesics is not limited to the declarative system or that the task requires some use of the declarative system in normal controls and, therefore, dis- 
advantages the amnesics. However, the multiple-systems account does not make an a priori prediction of a categorization deficit in amnesia.

2 . These power calculations are based on Cohen's (1988) conventions for effect size (i.e., small $d=.2$, medium $d=.5$, and large $d=.8$ ).

3 . Note that the approach taken here with regard to using only one outcome per published report is relatively conservative (Matt \& Cook, 1994). However, the issue of statistical independence of the data is further complicated in this meta-analysis. Because amnesic patients are so rare, they are often tested and retested in a number of these studies. Therefore, the lack of statistical independence of these studies is almost certain. Although there are statistical approaches in which dependencies between the data sets can be modeled (Matt \& Cook, 1994), these approaches require information that is often missing or unavailable in the original reports. Nonetheless, these are the data that have been used to claim a lack of a categorization impairment in amnesia, and so it is reasonable to test this claim on this data set.
4. Note that if the two results are meta-analytically combined in either of these cases, they yield a slight advantage for the control group over the amnesic group. Therefore, this double-counting is a more conservative approach.

5. In calculating the effect sizes, the most general available index of accuracy in the task was used. One exception to this was the data from probabilistic classification tasks. For these tasks, the argument has been made that amnesics eventually show deficits in categorization after prolonged training because controls can augment categories in their procedural system with items from declarative memory (Knowlton et al., 1996). Therefore, only data from the early learning stages in the probabilistic categorization tasks were used.

(Manuscript received October 13, 2003; revision accepted for publication January 28, 2004.) 